\section{Nuclear RNAi in fly}

Post-transcriptional gene silencing by RNA-mediated interference (RNAi) is well documented, but the role of RNAi components in transcriptional control in metazoans has remained elusive. Orlando and colleagues now show that RNAi components Dicer 2 (DRC2) and Argonaute 2 (AGO2) from Drosophila melanogaster associate with chromatin, with a strong

preference for transcriptionally active, euchromatic loci, including the heat-shock gene Hsp70. Knockdown of either DRC2 or AGO2 resulted in a significant increase in $H s p 70$ transcripts in cells that were not heat shocked. Chromatin immunoprecipitation (ChIP) analysis of DCR2depleted cells indicated decreased promoter-proximal pausing of RNA polymerase II (Pol II) on Hsp70 and decreased levels of NELF-E protein, which is part of the regulatory complex that causes Pol II pausing. These effects might be explained by the observed associations of DCR2 and AGO2 with Pol II and NELF-E. During heat shock, elongating Pol II undergoes dynamic repositioning from euchromatic regions to heatshock loci. Relocation of Pol II was repressed in Ago2 or Dcr 2 null mutant chromosomes, suggesting that AGO2 and DCR2 are required for global transcriptional repression after heat shock. Deep sequencing of AGO2associated small RNAs revealed strong enrichment for small RNAs that encompass the promoters as well as other regions of heat-shock loci and of most active genetic loci on both strands, but with a strong bias for the antisense strand, specifically after heat shock. Although the precise nuclear RNAi pathway in flies remains to be determined, the current data suggest that crucial RNAi components can contribute to transcriptional control by influencing the processivity of Pol II, thus revealing another level of transcriptome control. (Nature doi:10.138/nature10492, published online 6 November 2011)

$\mathrm{AH}$

\section{Itch (and Cyld) stops inflammation}

Proinflammatory cytokines such as TNF, IL-1 $\beta$ and IL-6 produced by tumor-associated macrophages can support tumor growth and metastasis. Receptor signaling from inflammatory stimulation leads to K63-linked polyubiquitination of the protein kinase Tak1 and activation through autophosphorylation. Activated Tak1, in turn, phosphorylates IKK, leading to downstream activation of NF- $\mathrm{KB}$-regulated genes. The E3 ligase Itch is a regulator of inflammation and the deubiquitinating enzyme Cyld regulates various human cancers and cytokine secretion in inflammatory cells. Cyld has been shown to cleave the K63-linked polyubiquitin chain on Tak1. Now, Venuprasad and colleagues show that Itch and Cyld work in concert to regulate the inflammatory response. They find that Itch or Cyld deficiency leads to growth and metastasis of lung carcinomas in inoculated mice, and the tumors have higher levels of TNF, IL-1 $\beta$ and IL-6. The authors show that Itch and Cyld form a complex that is able to remove the K63-linked polyubiquitin chain and exchange it for a K48-linked polyubiquitin chain, leading to proteasomal-mediated degradation of Tak1. In Itch- or Cyld-deficient macrophages, there was prolonged Tak1 activation and increased secretion of proinflammatory cytokines. Additional data indicate that the deubiquitinating activity of Cyld and its association with Itch are essential for terminating the Tak1mediated inflammatory response. As chronic cytokine production has been linked to tumor progression, Tak1 could be a new target for cancer treatment. (Nat. Immunol. 12, 1176-1183, 2011)

$M M$

Written by Inês Chen, Arianne Heinrichs, Steve Mason \& Michelle Montoya

\section{Signaling for purines}

Essential for synthesis of DNA and RNA and for cell growth, purine biosynthesis is facilitated by the reversible assembly of the purinosome, a large enzyme complex regulated by casein kinase 2 . Despite their integral role in cell behavior, it is not known how $\mathrm{G}$ protein-coupled receptors (GPCRs) contribute to purine biosynthesis. Now, using a cell-based dynamic mass redistribution (DMR) assay that measures redistribution of cellular material, Benkovic, Fang and colleagues have shown that $\mathrm{G}_{\mathrm{i}}$-coupled receptors have a role in regulating purinosome assembly. Assaying in HeLa cells, the authors screened 113 GPCR agonists for their ability to modulate small molecule-induced effects on purinosome assembly. Five adrenergic receptor agonists had an effect, and further characterization of their activity revealed that $\alpha_{2 \mathrm{~A}} \mathrm{AR}$, but not $\beta_{2}-A R$, is the receptor involved. Furthermore, purinosome assembly stimulated by $\alpha_{2 \mathrm{~A}}$-AR is mediated by $G \alpha_{i}$ activation, as inhibiting $\mathrm{G} \alpha_{i}$ with pertussis toxin (PTX) blocked the effects of the $\alpha$-AR agonist oxymetazoline. The ability of $G \alpha_{i}$ to mediate purinosome assembly extends beyond adrenergic receptors, as P2Y, LPA and prostaglandin receptor agonists all promoted purinosome formation in a PTX-sensitive fashion. Although many of the other proteins involved remain to be determined, the authors conclude that $\mathrm{G \alpha}_{\mathrm{i}}$ mediates a GPCR-driven pathway regulating purine biosynthesis, and GPCRs may represent a new class of targets for inhibiting this process. (Nat. Chem. Biol. 7, 909-915, 2011)

$S M$

\section{If yeast had brains}

Accumulation of the amyloid beta $(A \beta)$ peptide in the brain is the hallmark of Alzheimer's disease (AD), with evidence indicating that oligomeric forms of $A \beta$ are toxic to neurons, but the exact mechanisms are still unclear. $A \beta$ is generated from its precursor APP in the secretory pathway and reaches the extracellular space, where it interacts with the plasma membrane and undergoes endocytosis and vesicular trafficking. Genetic studies have pointed to endocytic pathway factors as risk factors for sporadic $A D$, but whether such effects are linked to $A \beta$ was unknown. Using the power of yeast genetics, Lindquist and colleagues screened for factors that modify the toxicity of $A \beta$. To mimic the fate of $A \beta$ in neurons, the authors fused $A \beta$ to a signal sequence, resulting in cleavage within the endoplasmic reticulum and secretion. $A \beta$ then interacted with the plasma membrane and was endocytosed. Importantly, the secreted $A \beta$ caused cellular toxicity. This approach uncovered 12 modifiers of $A \beta$ toxicity with clear human orthologs. Of these, three are involved in clathrinmediated endocytosis, and their human orthologs are known risk factors for AD. Seven are associated with cytoskeleton functions, and reexamination of previous clinical studies revealed a suggestive association of three of them with susceptibility to $A D$. Furthermore, the secreted $A \beta$ increased the number of clathrin foci and perturbed the localization of a plasma membrane protein that is normally endocytosed and trafficked to the vacuole. Finally, the authors validated the effects of some of these factors on $A \beta$ neurotoxicity using Caenorhabditis elegans and rat cortical neurons. Altogether, their work indicates that $A \beta$ can exert toxicity by disturbing neuronal endocytic homeostasis and highlights the value of yeast as a model system to identify novel players and provide mechanistic insights into a complex human disease. (Science doi:10.1126/science.1213210, published online 27 October 2011) 